\title{
A novel approach to predict fretting fatigue crack initiation
}

\author{
Guillaume Rousseau ${ }^{1,2, *}$, Claudio Montebello ${ }^{2}$, Yoann Guilhem ${ }^{1}$, and Sylvie Pommier ${ }^{1}$ \\ ${ }^{1}$ Ecole normale supérieure Paris-Saclay, 94230 Cachan, France \\ ${ }^{2}$ Safran Aircraft Engines, 77550 Moissy-Cramayel, France
}

\begin{abstract}
This paper proposes a simplified approximation of the velocity fields close to the contact edge using non-local intensity factors and reference fields. This non-local description is not affected by size or gradient effects and leads to unique crack initiation boundaries. Linear intensity factor $I$ represents the contribution of the elastic field on the total one and can be determined thanks to the macroscopic load even with coarse mesh. While the complementary intensity factor $\boldsymbol{I}^{\boldsymbol{c}}$ describes the friction effect and is proportional to the size of the partial slip zone. The prediction of $\boldsymbol{I}^{\boldsymbol{c}}$ using $\boldsymbol{I}$ through an incremental approach allows to predict the velocity fields for complex loadings and can be used to set up criteria to predict crack initiation.
\end{abstract}

\section{INTRODUCTION}

Fretting-fatigue occurs in engineering assemblies subjected to vibration loads. Micro-slip caused by oscillating forces, between the contacting bodies, leads to surface damage, and then crack initiation. Combined with cyclic bulk fatigue loading, crack propagation happens. Consequently, fretting-fatigue is a frequent source of failure constituting a major concern in safetycritical industries such as aerospace. Experimental test campaigns have shown that this phenomenon has an important negative effect on the material fatigue limit [1] due to the rise of embryo cracks very early in life.

One of the major difficulties in dealing with the fretting fatigue problem is the severe stress gradient close to the surface. Local approaches used to predict the fatigue life based on stress fields at the hot spots are over-conservative for fretting-fatigue because they do not account for the possibility of crack arrest due to a strong stress gradient from the surface to the bulk. Moreover, the crack initiation threshold is not unique in terms of local quantities (Fig. 1) and shows a strong dependency to the geometry [2-3]. However, using the theory of critical distance [4], it is possible to predict crack arrest and consequently to estimate fretting fatigue life and the gradient effect with a good degree of accuracy $[3,5]$.

In spite of the good predictions of fretting-fatigue threshold obtained using the theory of critical distance, there are criticisms with respect to the use of critical length as a material property [6]. Thus, the transferability of results obtained on laboratory samples (cylinder-plane contact) to industrial cases (disk-blade interface), which are complex and 3D geometry, is not guaranteed.
The goal of this paper is to propose a solution to predict the velocity field close to the contact edge by means of non-local intensity factors, independent from geometry, and to use these factors to set up a crack initiation criterion.

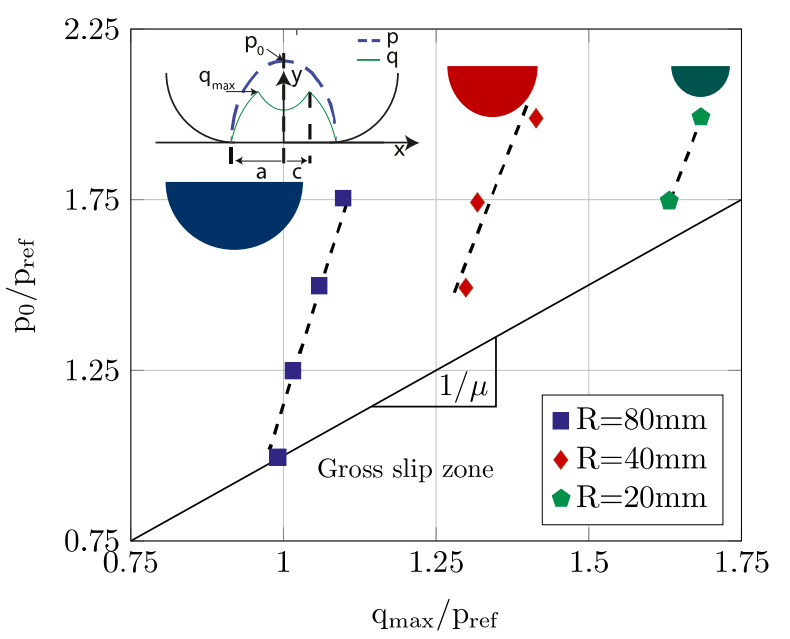

Fig. 1. Experimental crack initiation for Inconel 718 in plain fretting [2].

\section{MODELING}

\subsection{Background}

The interest of this approach, developed by Montebello [7] is shown in Fig. 2 where the description of the crack initiation boundaries through non-local intensity factors shows a unique threshold. Hence the 
geometry and the gradient effect have no effect on these variables.

In this part, the computation of the reference fields and the related intensity factors will be explained and an extension to fretting-fatigue problem will be made.

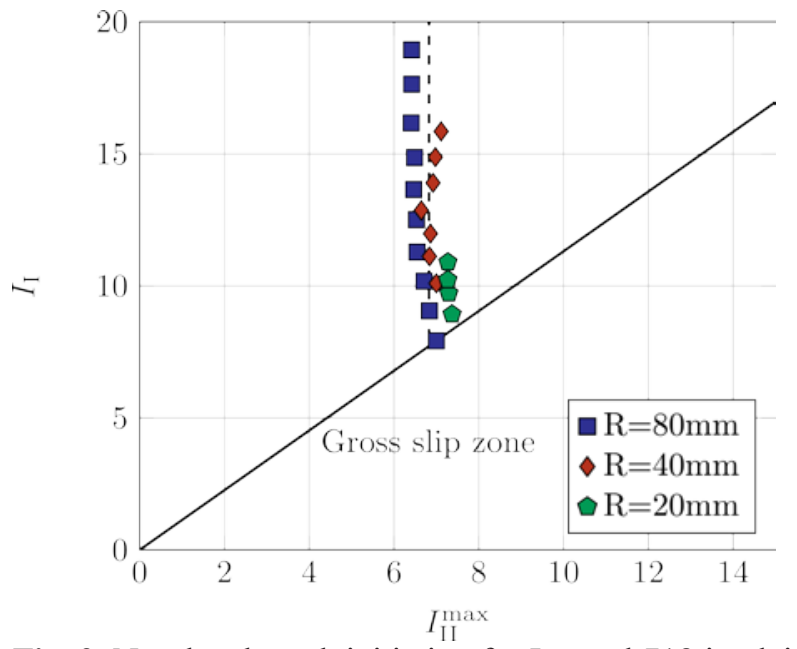

Fig. 2. Non-local crack initiation for Inconel 718 in plain fretting [8].

\subsection{Mechanical field partitioning}

Analogous to facture mechanics [9], a local self-similar geometry and a strong stress gradient induced by the contact, makes the spatial distribution of the mechanical fields imposed by the geometry while its intensity is determined by the macroscopic loads. Consequently, in the coordinate system $R^{\prime}$ attached to the contact edge (see Fig. 3) where $\boldsymbol{P}$ is the position coordinates vector, $I_{\mathrm{I}}$ and $I_{\mathrm{II}}$ the elastic intensity factors, $I_{\mathrm{II}}^{c}$ the complementary intensity factor and $\phi_{I}, \phi_{I I}$ and $\phi_{I I}^{c}$ the associated reference fields. The velocity field can be expressed through a sum of products between an intensity factor (time dependent) and a reference field (geometry dependent):

$$
\begin{gathered}
\boldsymbol{v}_{\boldsymbol{e}}(\boldsymbol{P}, t)_{R^{\prime}} \simeq I_{\mathrm{I}}(t) \boldsymbol{\phi}_{\mathrm{I}}(\boldsymbol{P})+I_{\mathrm{II}}(t) \boldsymbol{\phi}_{\mathrm{II}}(\boldsymbol{P}) \\
\boldsymbol{v}_{\boldsymbol{c}}(\boldsymbol{P}, t)_{R^{\prime}} \simeq I_{\mathrm{II}}^{c}(t) \boldsymbol{\phi}_{\mathrm{II}}^{c}(\boldsymbol{P}) \\
\boldsymbol{v}(\boldsymbol{P}, t)_{R^{\prime}} \simeq \boldsymbol{v}_{\boldsymbol{e}}(\boldsymbol{P}, t)_{R^{\prime}}+\boldsymbol{v}_{\boldsymbol{c}}(\boldsymbol{P}, t)_{R^{\prime}}
\end{gathered}
$$

This field is partitioned into two terms: the elastic one $v_{e}$ (Eq. 1) represents the elastic response of the structure; the complementary one $\boldsymbol{v}_{\boldsymbol{c}}$ (Eq. 2) describes the non-linear contribution of the friction in the slip zone. In turn $\boldsymbol{v}_{e}$ is separated into symmetric and antisymmetric parts with respect to the contact plane, analogous to mode I and mode II in LEFM. These fields are respectively generated by a variation of normal and tangential loads. Then the intensity factors are computed by projecting the velocity field $v$ to the reference field $\phi_{*}$ :

$$
\dot{I}_{*}(t)=\frac{\int_{\Omega} \boldsymbol{\nu} \cdot \boldsymbol{\phi}_{*}}{\int_{\Omega} \boldsymbol{\phi}_{*} \cdot \boldsymbol{\phi}_{*}}
$$

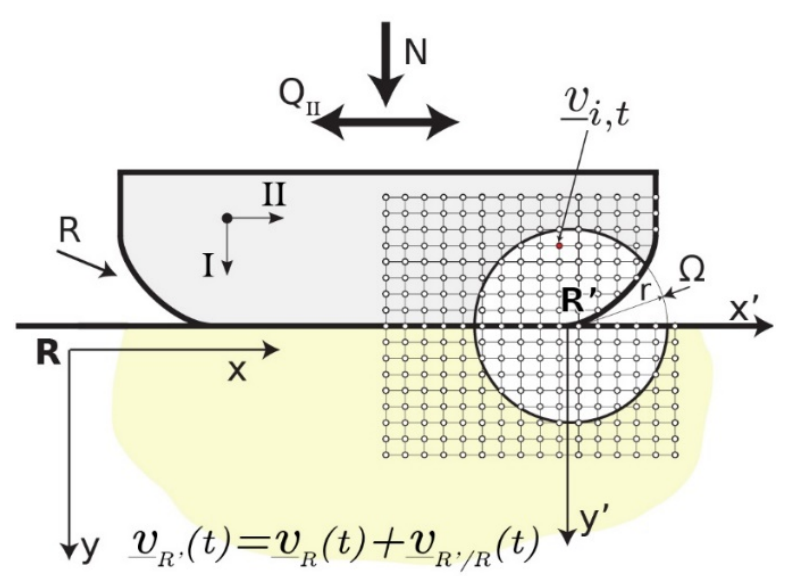

Fig. 3. FE parameters and extraction area.

\subsection{Extension to fretting fatigue}

The previous modelling is only usable for plain fretting because the fatigue loading $\sigma_{F}$ cannot be taken into account. Fig 4 (F-el and F-tot) shows the fretting modelling is unable to describe the velocity field. To solve this, the elastic velocity field must be enriched (Eq. 5) by an additional term. This termn takes into account the residual velocity field from the fatigue loading which cannot be described by the other intensity factors. The Karhunen-Loeve decomposition [10] is used to partition the residual field between two functions depending separately on time $I_{F}$ and space $\boldsymbol{\phi}_{\boldsymbol{F}}$.

$$
v_{\boldsymbol{e}}(\boldsymbol{P}, t)_{R^{\prime}}=\boldsymbol{v}_{\boldsymbol{e}}^{\text {Fretting }}(\boldsymbol{P}, t)_{R^{\prime}}+I_{F}(t) \phi_{\boldsymbol{F}}(P)
$$

\subsection{Quality of the assumption}

The final approximation of the velocity field for frettingfatigue is obtained (Eq. 3) and the elastic (Eq. 6) and total (Eq. 7) error of the approximation are defined.

$$
\begin{gathered}
\xi_{e l}=\frac{\sqrt{\int_{\Omega}\left(\boldsymbol{v}-\boldsymbol{v}_{\boldsymbol{e}}\right)^{2}}}{\sqrt{\int_{\Omega} \boldsymbol{v}^{2}}} \\
\xi_{\text {tot }}=\frac{\sqrt{\int_{\Omega}\left(\boldsymbol{v}-\boldsymbol{v}_{\boldsymbol{e}}-\boldsymbol{v}_{\boldsymbol{c}}\right)^{2}}}{\sqrt{\int_{\Omega} \boldsymbol{v}^{2}}}
\end{gathered}
$$


The error evolution during a proportional frettingfatigue cycle is shown in Fig. 4: the errors defined with the fretting modelling (F-el and F-tot) remains high. However, with the introduction of the fatigue field (Eq. 5), the total error remains low. It is worth noting that the elastic and total errors are equal when the whole contact is stuck.

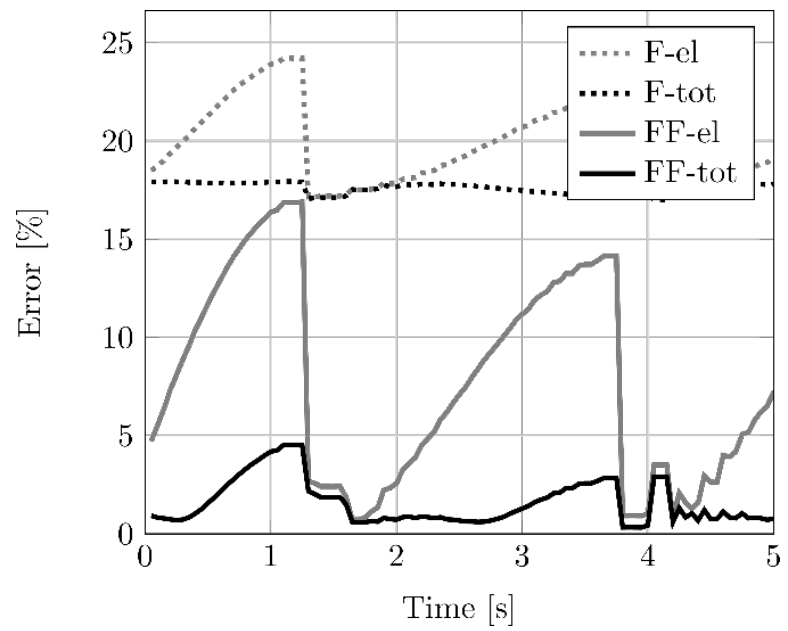

Fig. 4. Error evolution during a fretting-fatigue cycle with the plain fretting $(\mathrm{F})$ and the fretting-fatigue $(\mathrm{FF})$ modeling.

The additional fatigue intensity factors provide a good approximation of the velocity field in all the extraction area. However to characterize crack initiation, using the intensity factors from fretting is sufficient: different fatigue loadings imply different Wöhler curves (see Fig. 5), but when the intensity factor $I_{I I}$ is used to plot the Wöhler curve, a unique crack boundary is highlighted (see Fig 6).

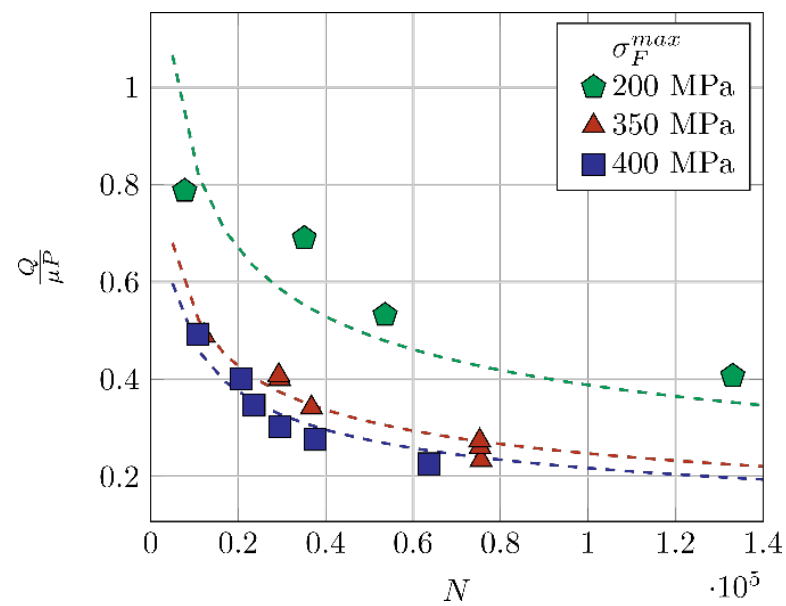

Fig. 5. Experimental Wöhler curve for fretting-fatigue crack initiation for Ta6V [11]

Fig. 2 and Fig. 6 show that linear intensity factors can be used to describe a unique crack initiation boundary for fretting and fretting-fatigue problem. These factors are directly linked to the macroscopic loading, so they can be computed with a coarse mesh FE simulation. The prediction of the non-linear intensity factor (which needs a fine mesh) thanks to the linear ones can lead to a good approximation of the velocity field close to the contact edge even with a coarse mesh.

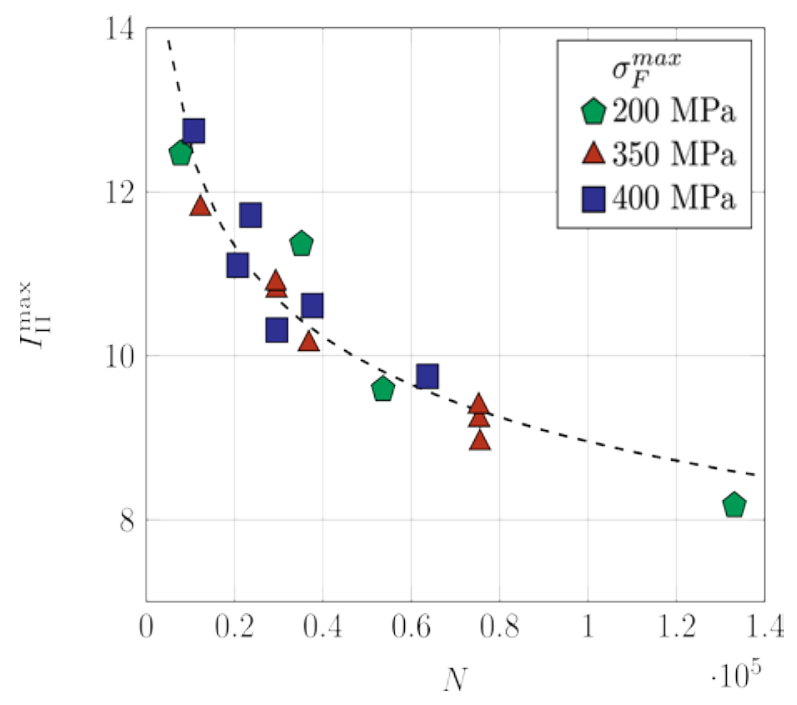

Fig. 6. Non-local Wöhler curve for fretting-fatigue crack initiation for Ta6V.

\section{INCREMENTAL MODEL}

The prediction of the non-linear intensity factor $I_{\mathrm{II}}^{c}$ will be made through an incremental approach which will be easy to use for complex loadings.

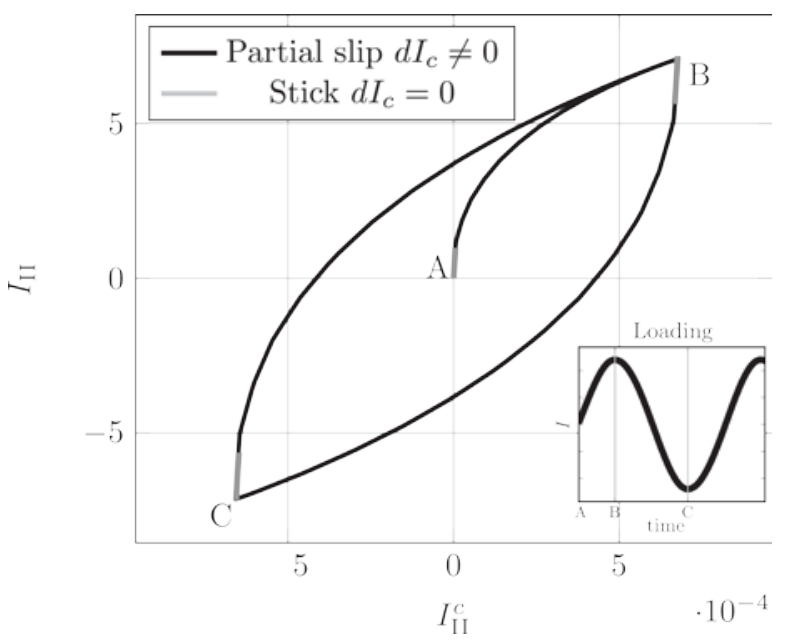

Fig. 7. Evolution of the intensity factors during a fretting cycle.

To model the non-linear behaviour (see Fig 7), a bilinear kinematic hardening law is chosen to provide a simple model along with a good precision. This law allows to predict the evolution of $I_{\mathrm{II}}^{c}$ during a fretting cycle with a complex tangential amplitude (see Fig. 8). 
To estimate more precisely the error, the complementary velocity field predicted by the model (Eq. 8) and the error associated (Eq. 9) are defined.

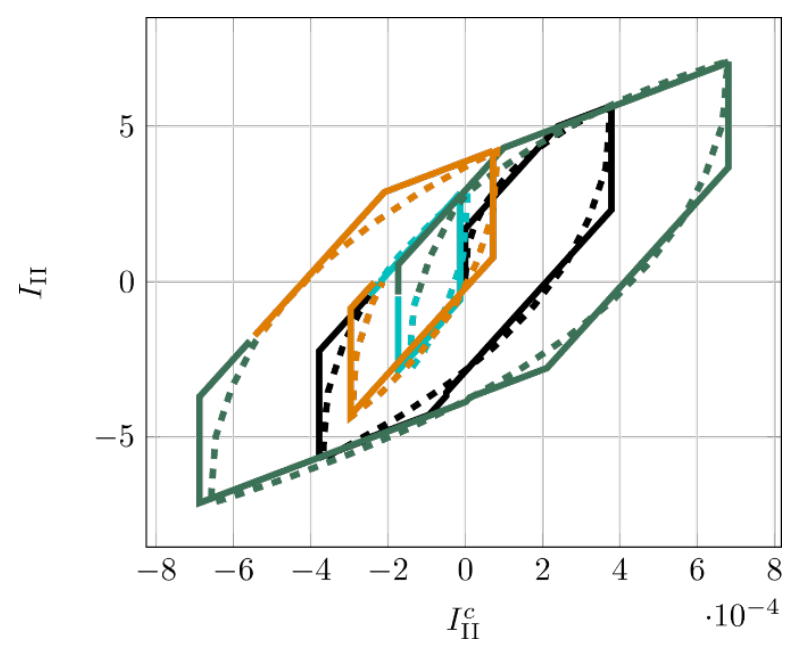

Fig. 8. Sliding prediction during a proportional fretting-fatigue cycle with variable amplitude.

$$
\begin{gathered}
\boldsymbol{v}_{\boldsymbol{c}}^{\text {model }}=\dot{I}_{I I}^{c^{\text {model }}(t) \boldsymbol{\phi}_{I I}^{c}(\boldsymbol{P})} \\
\xi_{\text {model }}=\frac{\sqrt{\int_{\Omega}\left(\boldsymbol{v}-\boldsymbol{v}_{\boldsymbol{e}}-\boldsymbol{v}_{\boldsymbol{c}}^{\text {model }}\right)^{2}}}{\sqrt{\int_{\Omega} \boldsymbol{v}^{2}}}
\end{gathered}
$$

With regard to the error on the velocity fields (Fig. 9), it appears that the complementary field predicted by the model permit to reduce the error, not as much as the complementary field issue from the simulation but to a reasonable level.

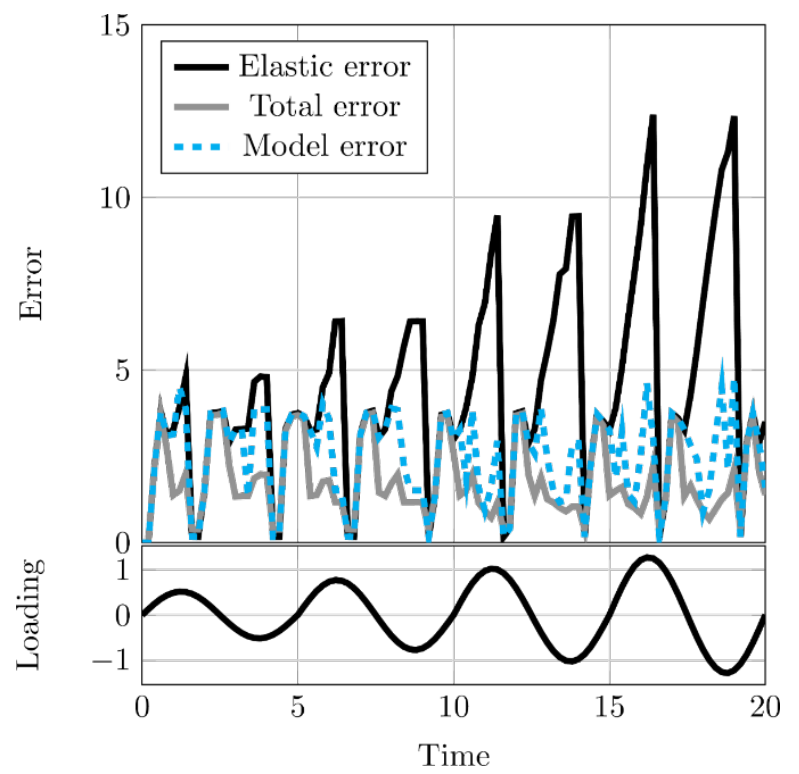

Fig. 9. Error evolution introduced by the approximation during a proportional fretting- cycle.

\section{CRACK INITIATION CRITERIA}

The previous parts highlighted a modelling allowing to plot unique crack initiation boundary for fretting but also for fretting-fatigue whereas the incremental model can predict the sliding $I_{\mathrm{II}}^{c}$ with $I_{\mathrm{II}}$. The aim of this part is to predict the crack boundaries using the intensity factors and to determine a material property characterizing the crack initiation threshold.

\subsection{Criteria}

To set up a criterion, non-local intensity factors will be used: (i) $d I_{I I}^{c}$ which is directly linked to the size of the partial slip zone; (ii) $I_{I I}$ which is proportional to the shear stress. This can be demonstrated using the analytical solution from LEFM [12] for mode I and II. Only three criteria are taken into account here but others can be formulated with these intensity factors.

Experiments are made with constant loading amplitude, so only one cycle is considered. Moreover, the spatial distribution of the damage is not taken into account because the contact front remains motionless and the maximum damage appears in the same area.

\subsubsection{Total sliding}

The first criterion is based on the accumulation of the micro sliding $d I_{I I}^{c}$ (the non-linear variable).

$$
C_{1} \sim \int_{t}\left|d I_{I I}^{c}\right|
$$

\subsubsection{Energy dissipated by friction}

The second criterion is based on the energy dissipated by friction on the contact surface.

$$
C_{2} \sim \int_{t}\left|I_{I I} d I_{I I}^{c}\right|
$$

\subsubsection{Energy release rate}

The last criterion is analogous to the energy release rate in LEFM and express the hypothesis that failure occurs when the mechanical power reaches a critical value.

$$
C_{3} \sim \max \left(I_{I I}^{2}\right)
$$




\subsection{Methodology}

1. The experimental works highlight some crack initiation frontiers expressed in $\left(\mathrm{q}_{\max }-\mathrm{p}_{0}\right)$ or $(\mathrm{Q} / \mu \mathrm{P}-\mathrm{N})$ coordinates. For each point on these frontiers the relative macroscopic loads are computed.

2. A FE computation, using the macroscopic loads calculated previously, is performed to obtain the relative velocity field close to the contact edges.

3. The non-local intensity factors are computed.

4. An average value of each criterion is computed through all the data.

5. Non-local (and consequently local) quantities are predicted thanks to the criteria and a crack initiation map is built.

It is worth mentioning that experimental data are enriched; a linear interpolation of the experimental data point is done. This permits to apply the steps listed above on a more complete set of data.

\subsection{Results}

The three criteria have been processed with the previous experiments to evaluate their ability to predict crack initiation boundary for constant and variable lifetimes.

\subsubsection{Constant lifetime}

The non-local boundary is predicted with the methodology describes in section 4.2 and the inverse post-treatment is applied to plot the local map (Fig. 10)

The three criteria are able to predict boundaries for the highest radii $(40$ and $80 \mathrm{~mm}$ ). The results are less satisfactory for the smallest radius, where criteria 2 and 3 respectively overestimate and underestimate the boundary. Concerning the criterion 1 , it seems unable to predict correctly the boundary.

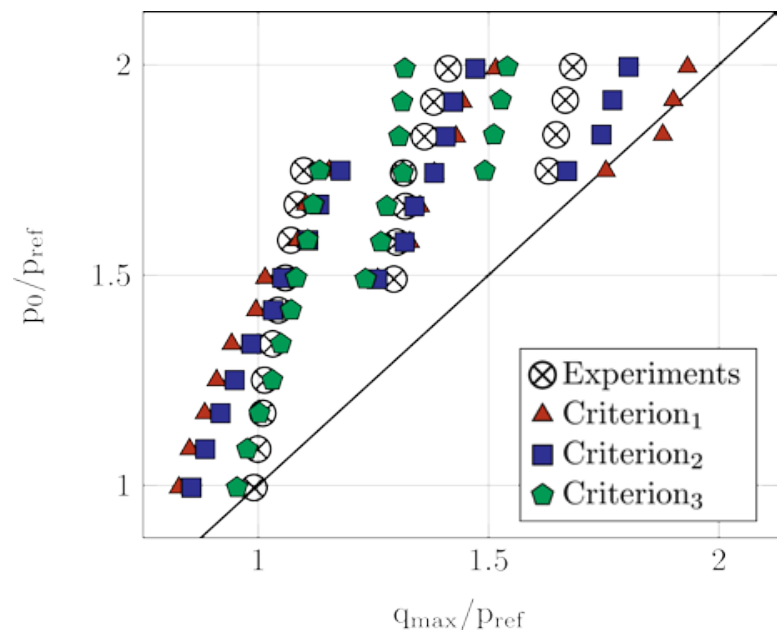

Fig. 10. Local crack initiation boundary predicted by the criteria for Inconel 718.

\subsubsection{Variable lifetime}

The three criteria are able to predict the boundaries when the lifetime is higher than 20000 cycles. Until this limit, only the criteria based on the dissipated energy by friction gives good results. However, for short lifetime, stress is high and plasticity may not be neglected.

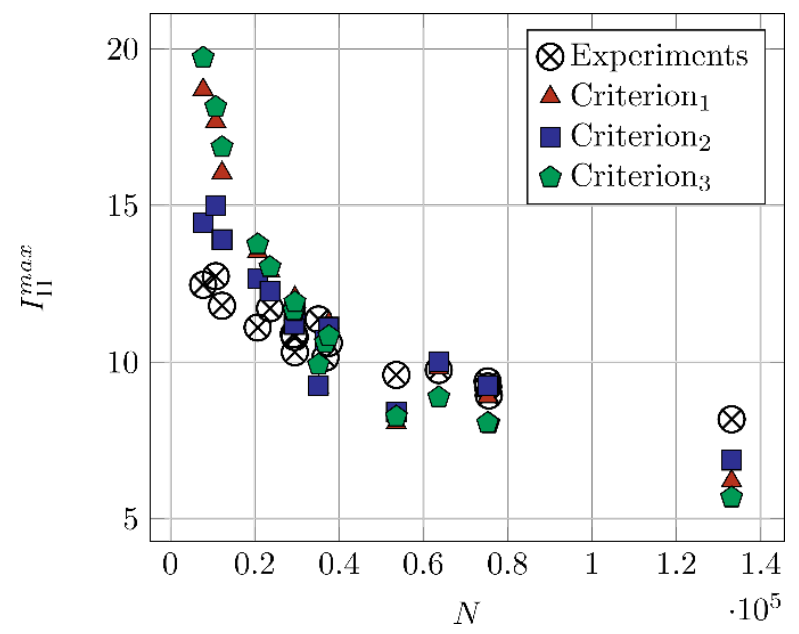

Fig. 11. Non-local crack initiation boundary predicted by the criteria for Ta6V.

\section{3D MODELING}

The contact between disc and root blade corresponds to a complex geometry and is submitted to non-proportional loading. Consequently, a 3D modelling is required to study such industrial cases. The fretting modelling (Eq. 2) can be extended in 3D with the addition of two new intensity factors and associated reference fields: (i) a linear one $I_{I I I}$ to take into account the out of plane displacement (Eq. 13); (ii) a complementary one $I_{I I I}^{c}$ (Eq. 14) to describe the friction effect in this new direction.

$$
\begin{gathered}
v_{e}(\boldsymbol{P}, t)_{R^{\prime}} \simeq I_{\mathrm{I}}(t) \boldsymbol{\phi}_{\mathrm{I}}(\boldsymbol{P})+I_{\mathrm{II}}(t) \boldsymbol{\phi}_{\mathrm{II}}(\boldsymbol{P}) \\
+I_{\mathrm{III}}(t) \boldsymbol{\phi}_{\mathrm{III}}(\boldsymbol{P}) \\
\\
v_{c}(\boldsymbol{P}, t)_{R^{\prime}} \simeq+I_{\mathrm{II}}^{c}(t) \boldsymbol{\phi}_{\mathrm{II}}^{c}(\boldsymbol{P}) \\
+I_{\mathrm{III}}^{c}(t) \boldsymbol{\phi}_{\mathrm{III}}^{c}(\boldsymbol{P})
\end{gathered}
$$

The incremental model introduced previously is still able to predict $\left(I_{I I}^{c}, I_{I I I}^{c}\right)$ using $\left(I_{I I}, I_{I I I}\right)$ for complex nonproportional tangential loading conditions. Fig. 12 shows the different errors under non-proportional tangential loading which remains low for the model.

However, the lack of experiments with complex and non-proportional loadings makes the identification of criterion difficult. 


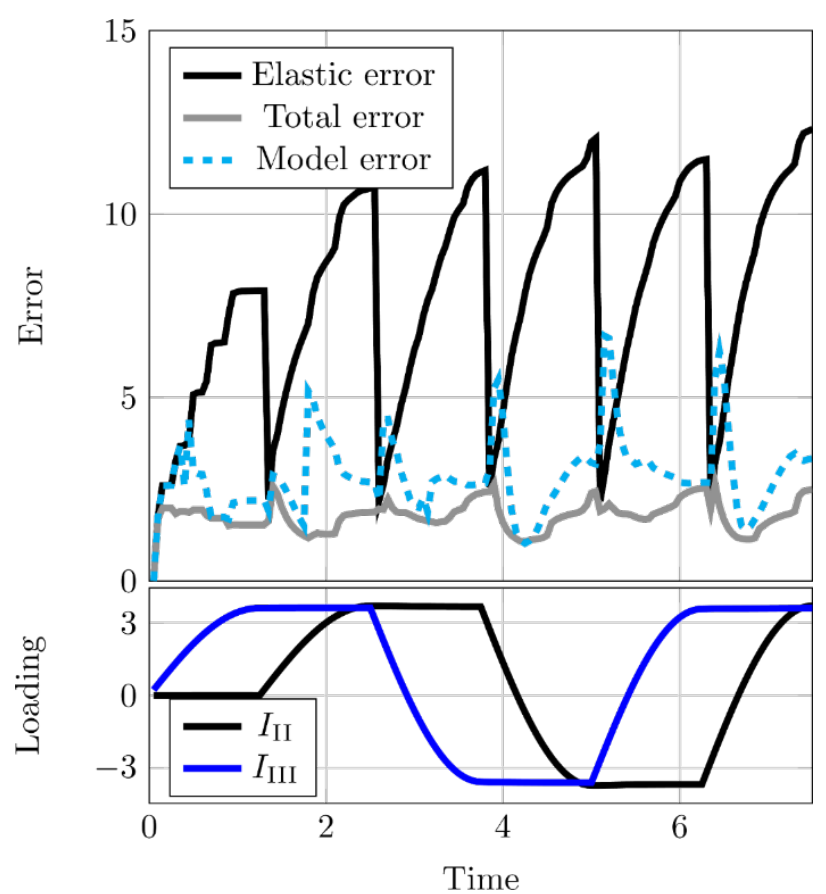

Fig. 12. Errors evolution introduced by the approximation during a non-proportional fretting-fatigue cycle.

\section{CONLUSION}

A novel approach to predict the local velocity field of a fretting-fatigue case, based on a fracture mechanics analogy, has been presented. Once the reference fields are identified for a specific geometry, it is possible to predict the velocity field close to the contact edge thanks to the non-local intensity factors directly from the macroscopic loadings. Finally, using a criterion based on the energy dissipated by friction on the contact surface, the crack initiation boundary can be predicted. Moreover, as the size and gradient effects are included in the reference fields, the use of criteria and laws based on non-local intensity factors are not geometry dependent. Consequently, they can be identified on laboratory tests and easily transferred to industrial structure.

The displacement of the contact edge and the damage accumulation should be taken into account to study normal load variations and all kinds of complex loadings. To complete this work, fretting tests in nonproportional and variable amplitude could be performed to verify the results obtained by simulation.

The authors are grateful to Safran Aircraft Engines (Safran group) for its financial support within the IRG COGNAC project framework.

\section{REFERENCES}

1 TC Lindley. Fretting fatigue in engineering alloys. Int J Fatigue 19:39-49 (1997).

2 R Ferre, S Fouvry, B Berthel, JA Ruiz-Sabariego. Stress gradient effect on the crack nucleation process of a Ti-6Al-4V titanium alloy under fretting loading: Comparison between non-local fatigue approaches. Int J Fatigue 54:56-67 (2013).

3 R Amargier, S Fouvry, L Chambon, C Schwob, C Poupon. Stress gradient effect on crack initiation in fretting using a multiaxial fatigue framework. Int J Fatigue 32:1904-12 (2010).

4 D Taylor. The Theory of Critical Distances: Basics. Eng Fract Mech 75:1696-705 (2008).

5 JA Araújo, L Susmel, D Taylor, JCT Ferro, EN Mamiya. On the use of the Theory of Critical Distances and the Modified Wöhler Curve Method to estimate fretting fatigue strength of cylindrical contacts. Int J Fatigue 29:95-107 (2007).

6 FC Castro, JA Araújo, N Zouain. On the application of multiaxial high-cycle fatigue criteria using the theory of critical distances. Eng Fract Mech 76:512-24 (2009).

7 C Montebello. Analysis of the stress gradient effect in Fretting-Fatigue through a description based on nonlocal intensity factors. Université Paris-Saclay, 2015. 8 C Montebello, S Pommier, K Demmou, J Leroux, J Meriaux. Analysis of the stress gradient effect in frettingfatigue through nonlocal intensity factors. Int. J. Fatigue, vol. 82, Elsevier; , p. 188-98.

9 A. E Giannakopoulos, TC Lindley, S Suresh. Aspects of equivalence between contact mechanics and fracture mechanics: theoretical connections and a lifeprediction methodology for fretting-fatigue. Acta Mater 46:2955-68 (1998).

10 M Loève. Probability Theory I 45:1-10 (1977).

$11 \mathrm{~J}$ Meriaux. Etude expérimentale de modélisation de l'endommagement d'un contact aube disque de soufflante soumis à des chargements de fretting fatigue. 2010.

12 HM Westergaard. Bearing pressures and cracks. J Appl Mech 61:A49-53 (1939). 